\title{
Mega sistema estructural Cajamarca, configuración estructural sugerida en relación con la mineralización de pórfidos y epitermales en el Norte del Perú
}

\author{
Mega structural system Cajamarca, structural construction suggested in \\ relation to the mineralization of porphyries and epithermals in northern Peru
}

\author{
Jesús A. Torres Guerra ${ }^{1}$, José Tumi Pacheco ${ }^{2}$, Mariel Samanez Trigoso ${ }^{2}$, Yordy Caycho Vilca ${ }^{2}$
}

Recibido: 01/07/2020 - Aprobado: 30/10/2020 - Publicado: 30/11/2020

\begin{abstract}
RESUMEN
El norte del Perú se caracteriza por la presencia de yacimientos tipo pórfido (Cu-Au, y Cu-Mo) y epitermales (Au) de clase mundial, ubicados en el sector occidental de los Andes centrales en el norte del Perú. Estas minas y depósitos mineralizados se presentan en relación temporo-estructural asociados a magmatismo del Neógeno con edades desde $24 \mathrm{Ma}$ a $7 \mathrm{Ma}$., intruyendo rocas silicoclásticas del Cretáceo superior, que por efectos de las deformaciones tectónicas particularmente en el Cenozoico (fases Incaica y Quechua), ocasionaron la ocurrencia de mega controles estructurales complejos como el que se propone en la siguiente publicación y espera abrir una ventana de debate en su entorno.

Estructuralmente, los yacimientos en el norte del Perú, están controlados por mega sistemas estructurales que contienen en el sector oriental como principal accidente estructural la falla Punre - Canchis (Rivera et al., 2008) así como los pliegues y fallas del sistema estructural del Marañón, y en el sector occidental incluyen fallas al este de Chongoyape que limitan el Batolito de la Costa hacia el borde oriental, así como los silicoclásticos del Mesozoico, poniéndolos en contacto con volcánicos del Cenozoico. La cuenca en su sector occidental habría sufrido cambios en los estados de esfuerzos regionales, produciendo movimientos epirogénicos, desde el Cretácico superior, jugando un importante rol las grandes fallas regionales controlando la cuenca extensiva a través de fallas normales (PardoiCasas \& Molnar, 1987). La subducción se dio de manera oblicua y con un bajo ángulo, generando así zonas permeables, de dimensiones kilométricas, generando mega estructuras paralelas y conjugadas que dieron lugar a jogs estructurales. Luego del análisis y evaluación de la información recopilada, nos permiten sugerir un nuevo concepto para definir el comportamiento estructural de este sector de los andes peruanos, proponiendo la denominación Mega Sistema Estructural Cajamarca, a un sistema de cinemática sinextral compresivo y de dirección andina, en el Mioceno, también consideramos que en este sector norte del país, se ha desarrollado una zona de convergencia oblicua de las placas en la zona de subducción, así como la variación del ángulo de subducción (15), como rasgos importantes para la creación de depósitos minerales. En este sentido, este concepto tiene como principal objetivo involucrar un macro mecanismo general de entendimiento e interpretación de conjunto, que involucran estudios estructurales antes realizados. Palabras clave: Exámenes médicos preocupacionales y periódicos; salud ocupacional; hipoacusia; planta de teñidos textil.
\end{abstract}

Palabras clave: Mega sistemas estructurales; dextral; sinestral; depósitos; fases inca y quechua.

\begin{abstract}
The north of Peru is characterized by the presence of world-class porphyry (Cu-Au and Cu-Mo) and epithermal (Au) deposits, located on the west of central Andes, northern Peru. These mines and mineralized deposits are present in a temporal-structural associated with Neogene magmatism with ages from $24 \mathrm{Ma}$ to $7 \mathrm{Ma}$, intruding silica-clastic rocks from the upper Cretaceous, which, due to the effects of tectonic deformations, particularly in the Cenozoic (Incaica and Quechua phases), led to the occurrence of mega controls Structural problems such as the one proposed in the following publication and hopes to open a window of debate in its environment.

Structurally, the deposits in the north of Peru are controlled by mega structural systems that contain the Punre - Canchis fault (Rivera et al., 2008) in the eastern sector as the main structural accident, as well as the system of folds and faults of the Marañón, and in the Western sector include faults to the East of Chongoyape that limit the eastern edge of the Batolito of the Coast, as well as the silicoclastics of the Mesozoic, putting them in contact with volcanos of the Cenozoic. The basin in its western sector would have undergone changes in the status of regional efforts, producing epirogenic movements, since the Upper Cretaceous, with large regional faults playing an important role, controlling the extensive basin through normal faults.

\footnotetext{
1 Docente de la Escuela Académico Profesional de Ingeniería Geológica de la Universidad Nacional Mayor de San Marcos. Lima, Perú Autor para correspondencia: albertorresg@hotmail.com ORCID: https://orcid.org/0000-0001-8186-5249 E-mail: iigeo@unmsm.edu.pe
}

2 Estudiante de la Escuela Profesional de Ingeniería Geológica de la Universidad Nacional Mayor de San Marcos. Lima, Perú.
\end{abstract}


(Pardo-Casas \& Molnar, 1987). The subduction occurred obliquely and with a low angle, thus generating permeable zones, of kilometer dimensions generating mega parallel and conjugate structures that gave rise to structural jogs. The analysis and evaluation of the compiled information allows us to suggest a new concept to define the structural behavior of this sector in the Peruvian Andes, proposing a new denomination Mega Cajamarca Structural System for a sinextral system with compressive behavior and with Andean direction in the Miocene. It is also considered that in this north sector, an oblique convergence plaque area has been developed in the subduction zone, as well as the subduction angle variation (15 degrees), as main characteristic for the mineral deposit generation. In this way, this concept has the principal objective to involve a general mechanism of an ensemble understanding and interpretation, including previously developed structural studies.

Keywords: Mega structural systems; dextral; sinestral; deposits; inca and quechua phases.

\section{INTRODUCCIÓN}

El presente artículo nace como una inquietud académica de revisar, analizar y reinterpretar información geológica disponible a la fecha con respecto a las características estructurales relacionadas con los procesos de formación de minerales en el norte del Perú, particularmente en la región Cajamarca. Consideramos importante analizar la acción de los campos de esfuerzos y sus implicancias en la explicación de manera conjunta de sus efectos y sus relaciones con la ocurrencia de mineralización económica de oro y cobre.

Esta investigación es parte del desarrollo correspondiente a la práctica en el curso de Yacimientos de Minerales Metálicos, de la Escuela Académico Profesional de Ingeniería Geológica, liderado por el Mg. Jesús A. Torres G. y conto con el valioso apoyo en la discusión, análisis e interpretación de los alumnos: Yordy Caycho, Mariel Samanez y José Tumi, del quinto año de estudios.

La finalidad fue incorporar conceptos nuevos en la interpretación de los campos de esfuerzos y sus efectos e implicancias en la generación de mega estructuras como el Mega Sistema Estructural Cajamarca, que se propone, es un concepto que nos puede ayudar en rediseñar y redireccionar nuestros esfuerzos de exploración hacia lugares que no hayan sido lo suficientemente investigados y que tengan un interesante potencial.

\section{ESTRATIGRAFÍA}

Como punto de partida de todo análisis geológico, es importante que tengamos control sobre la estratigrafía que domina en el área estudiada, abarcando principalmente la región Cajamarca, donde existen gran heterogeneidad de rocas y edades que nos evidencian una complicada historia geológica desde el Proterozoico hasta el Cuaternario. La geometría de las formaciones rocosas y su posición fue desarrollándose desde el Proterozoico, pasando por el Paleozoico, donde se formó el basamento que luego sustento la formación de rocas del Mesozoico y Cenozoico.

\subsection{Basamento Precámbrico - Paleozoico Inferior}

Entre los $6^{\circ} 00^{\prime}$ y $7^{\circ} 30^{\prime} \mathrm{S}$, que corresponde a la parte oriental de la región, afloran rocas metamórficas que se deduce se formaron entre le Proterozoico y el Paleozoico inferior, particularmente en San Marcos y Celendín. De acuerdo con el grado de metamorfismo se han diferenciado dos unidades litoestratigráficas, que se designan como el Complejo del Marañón del Neoproterozoico y el Complejo de Olmos del Paleozoico inferior. Los gneises y anfibolitas que conforman el Complejo del Marañón son parte de la cordillera Oriental. Cabe mencionar que, hacia la frontera con Ecuador, tanto en la cordillera del Cóndor como en la Occidental afloran rocas similares a ambos complejos.

Por otro lado, el Grupo Salas consiste en filitas pelíticas y pizarras que se intercalan con cuarcitas laminadas correspondiendo al Paleozoico inferior.

\subsection{Cobertura Mesozoica}

Como resultado de los movimientos orogénicos, así como su posterior ciclo de denudación continental se dio lugar a la formación Mitu de características continentales, que ocurre en la cordillera Oriental como consecuencia de la tectónica Hercínica.

Las rocas cretácicas del Grupo Goyllarisquizga cubren gran parte de la región, constituida por las formaciones Chicama, Chimú, Santa Carhuaz. Una gran parte de la secuencia estratigráfica está bien expuesta en el distrito Minero de Hualgayoc.

En Cerro Corona afloran sedimentitas del Cretácico de la Formación Chúlec, constituidas por margas y calizas nodulares, que infrayacen en forma concordante a Formación Pariatambo, constituidas por arcillitas y calizas bituminosas con estratos delgados, que infrayace en concordancia paralela y ondulante a la Formación Yumagual, constituida por margas y calizas gris parduzcas y destacando un nivel de arcillita margosa amarillenta.

En Minas Conga afloran calizas grises, arcillitas arenosas y margas delgadas de la Formación Quilquiñán; calizas nodulares, margas y arcillitas de la Formación Mujarrún; margas y calizas gris parduzcas de la Formación Yumagual; arcillitas y calizas bituminosas de la Formación Pariatambo; margas, limolitas y calizas nodulares de la Formación Chúlec; areniscas calcáreas y limolitas ferruginosas de la Formación Inca y cuarcitas y areniscas del Grupo Goyllarisquizga.

En Tantahuatay, la base de la columna litoestratigráfica está conformada por el Grupo Goyllarisquisga (cuarcitas y areniscas) del Cretácico inferior, al cual suprayacen las formaciones Inca, Chulec, Pariatambo y Grupo Pulluicana (silicoclásticos y carbonatos) del Cretácico superior. Las cuales son intruidas por monzonitas porfiríticas, luego ocurren tres etapas de actividad volcánica, constituidas por emisiones volcánicas a partir de fisuras, brechas-pipe y/o diatremas, siguen diques y domos andesíticos-dacíticos y finalmente aparecen chimeneas de brechas. 


\subsection{Cobertura Cenozoica}

A principios del Paleogeno o fines del Cretácico, ocurrió una tectónica, que dio lugar a un ciclo de denudación continental dándose las condiciones para la Formación Chota de características continentales, y luego la formación de las cuencas volcánicas del Paleógeno y Neógeno, siendo la Formación Llama compuesta de rocas piroclásticas de composición andesítica la más antigua en la cuenca volcánica de Cajamarca. La Formación Porculla del Mioceno, compuesta por derrames y brechas dacíticas de gran extensión en la región, suprayace a la Formación Llama e infrayace a la Formación Huambos del Neogeno.

En la mina La Zanja las rocas más antiguas corresponden con rocas clásticas - carbonatados del Cretáceo del Grupo Goyllarisquizga con fuerte plegamiento. Suprayace un complejo volcánico conformado por una caldera constituida por la Formación Porculla del Mioceno. Esta caldera está compuesta por ignimbritas soldadas y flujos piroclásticos dacíticos en la base, luego es cortada por domos andesíticos-dacíticos y riodacíticos-riolíticos. Adicionalmente se ha reconocido, tres centros volcánicos del Mioceno: La Zanja, Hueco Grande y San Miguel. Los depósitos recientes están conformados por depósitos fluviales, aluviales y fluvioglaciares.

\subsection{Magmatismo}

Los intrusivos del Mioceno están relacionados a la existencia de yacimientos de gran extensión correspondientes a la Franja de pórfidos de $\mathrm{Cu}-\mathrm{Mo}(\mathrm{Au})$. Esta franja presenta tres eventos magmáticos relacionados con la mineralización, que van desde los $22 \mathrm{Ma}$ hasta los $5 \mathrm{Ma}$ (Quispe et al., 2008).

El primer evento entre 22 y 20 ma, comprende los yacimientos de pórfido de $\mathrm{Cu}$-Mo de Michiquillay y Aurora Patricia.

El segundo evento entre 18 y $13 \mathrm{Ma}$, está relacionado con los pórfidos de Cu-Mo de El Galeno, La Granja, Cañariaco y los pórfidos de $\mathrm{Cu}-\mathrm{Au}$ en Minas Conga (Perol-Chailhuagon) y Cerro Corona, en este caso algunos intrusivos correspondientes a la edad de este evento, han conformado skarns y cuerpos de reemplazamiento de $\mathrm{Pb}-\mathrm{Zn}-\mathrm{Ag}$ en contacto con formaciones calcáreas del Cretácico, ejemplos son: Chungar, Iscaycruz datados en 13 Ma. Para el caso de El Galeno, Magistral y Pachagón, se han observado indicios de un emplazamiento sintectónico de los stocks de pórfidos de $\mathrm{Cu}$ durante los eventos del Mioceno. Por otro lado, otros sistemas porfiríticos similares a los citados, afloran localmente donde se ha erosionado la cobertura de los volcánicos del Mioceno (Franja XXII), guardando relación con los depósitos de Chamis, Colpayoc, Cascabamba, San José, La Arena, Alto Dorado, Pashpap, Los Latinos, así como el pórfido-epitermal El Toro y el skarn distal de Pb-Zn-Cu El Extraño (Quispe et al., 2008).

El tercer evento entre 10 y 5 ma está representado por el pórfido $\mathrm{Cu}-\mathrm{Mo}(\mathrm{Au})$ de Río Blanco en Piura, mientras que al sur de la franja se localizan los depósitos de Toromocho y Puy Puy. Los pórfidos de este tercer evento, en contacto con rocas carbonatadas del Cretácico desarrollaron skarn y cuerpos de reemplazamiento proximales $(\mathrm{Cu}-\mathrm{Zn})$ y distales
(Pb-Zn-Ag), como Antamina, Huanzalá, Pachapaqui, Raura, Ucchuchacua (puede ser más antigua), Huarón, Yauricocha, entre otros.

Con respecto a las rocas de caja, las areniscas y cuarcitas dominan gran parte del depósito El Galeno, así como en Michiquillay donde adicionalmente se tiene calizas. En Minas Conga sectores Chailhuagon y Perol se tienen limolitas calcáreas, arcillitas, calizas y margas nodulares que son las rocas caja.

El magmatismo del Paleógeno en la región de Cajamarca, entre 57 y $43 \mathrm{ma}$, se caracteriza por su composición intermedia con perfiles planos de tierras raras (REE) y primitiva composición de isótopos, lo que indica que provienen de un manto inmaduro dominado por piroxenos y olivinos (R. C. I. Davies, 2006). Esta variación magmática corresponde con el comienzo del sistema de pliegues y fallas que afectan a las secuencias sedimentarias por la tectónica andina fase incaica del Eoceno medio (Soler, 1991) ; (Skinner \& Benavides-Cáceres, 1999).

Los grandes sistemas de pórfidos se ven asociados a la mayor producción de magmas félsicos, como consecuencia del engrosamiento cortical de los Andes (Mamani et al., 2010), producto de la subducción, desde el periodo Paleógeno temprano, siendo los de franja de epiermales del Mioceno-Plioceno, los yacimientos de mayor extensión y contenido de $\mathrm{Au}$ como las minas: Yanacocha (11.9 $\mathrm{Ma})$, La Zanja (15.6 Ma), Tantahuatay (12.4 Ma), Minas Conga (15.6 Ma), entre otros, que a su vez se encuentran relacionados a centros volcánicos complejos, calderas, brechas y domos, albergados en rocas magmáticas de composición intermedia a félsica.

Por otro lado, el principal metalotecto aurífero en el norte del Perú está constituido por secuencias volcánicas del Grupo Calipuy del Mioceno, con un importante valor económico y para las exploraciones.

\subsection{Control Estructural y Mineralización}

El desarrollo de sistemas de cizalla en la tectónica Andina ha influenciado en gran parte a la localización de los controles estructurales y la mineralización en el norte del Perú, se plantea que genéticamente se relacionan con mega sistemas de fallas regionales de rumbo andino que en función de su convergencia oblicua respecto de las placas tectónicas tienen un factor de rumbo, formando así fallas de desplazamiento de rumbo tipo strike slip, lo que es claramente observable en los corredores estructurales San Pablo - Porculla (Enríquez et al., 2000) y Michiquillay - Hualgayoc. Con respecto al corredor Chicama Yanacocha, transversal al andina se explica considerando que estas son expresiones estructurales secundarias de las fallas de rumbo andino (Rivera \& Santisteban, 2008). Sin duda, los conceptos anteriores son válidos y se encuentra debidamente sustentados, razón por la cual es importante darle un marco estructural de conjunto.

En este sentido, se sugiere la denominación de Mega Sistema Estructural Cajamarca, que significa la configuración de un conjunto estructural en sí mismo y que a su vez define un marco estructural con límites que delimitan dicho conjunto. De esta manera, hacia 
el oriente tendremos un límite paralelo y al Este del sistema Punre - Canchis, relacionado con un sistema de fallas de tipo sinestral inverso y un límite occidental conjugado y relacionado con un sistema de fallas al Este de Chongoyape que limita a los silicoclásticos del Mesozoico y las estribaciones del Batolito de la Costa con las rocas del Cenozoico, estas son fallas de desplazamiento de rumbo y lineamientos estructurales que presentan una cinemática sinestral y no ofrecen una continuidad evidente puesto que se encuentran ocultas por los volcánicos de la Formación Llama del Cenozoico. Desde el punto de vista morfo estructural, se asemeja un mega cimoide donde se puede diferenciar los sistemas Riedel (1 y 2), las fallas P de tipo conjugadas e Y paralelas a la envolvente principal. El sistema de fallas sinestrales se habría originado a partir de una margen extensional (Cretáceo inferior), dando comienzo de esta manera a las diferentes cuencas tectosedimentarias (cuencas pull apart) en su interior. Durante etapas de compresión oblicua se formaron estructuras secundarias a la falla principal, de esta manera se da lugar así a zonas de dominio estructural como zonas de debilidad preparadas para el emplazamiento de los depósitos de tipo porfiríticos $\mathrm{Cu}-\mathrm{Mo}(\mathrm{Au})$ y epitermales $(\mathrm{Au}-\mathrm{Ag})$ en el Mioceno.

Correlaciones espaciales, temporales y de mineralización permiten sobreentender que el movimiento relacionado con la mineralización en los diferentes corredores estructurales no son los mismos. El corredor San Pablo-Porculla se localiza en la sección occidental del Mega Sistema Estructural Cajamarca, y se asocia a una cinemática de movimiento relativo de tipo sinextral, con variada morfología donde las vetas principalmente presentan cimoides, y colas de caballo, localmente desarrollan morfología anastomosada como en los Pircos, Lucero, Paredones y otros, constituye un excelente corredor estructural vinculado con mineralización epitermal de baja sulfuración, evidenciado en Los Pircos donde se localizó mineralización de oro de tipo bonanza.

Por otro lado, el corredor Michiquillay-Hualgayoc se localiza en el sector Oriental del Mega Sistema Estructural Cajamarca, y dada su ubicación su relación estructural es directa con el sistema de fallas Punre-Canchis (Rivera et al., 2008), que corresponde a un sistema de fallas regionales sinextrales inversas, que empezaron a formarse en el Cretáceo dando origen a cuencas tectónicas como es el caso de la de Cajamarca, que evolucionaron en el Cenozoico y generaron los espacios para la mineralización de pórfidos y epitermales (R. C. Davies \& Williams, 2005).

La acción de la convergencia oblicua entre la placa de Nazca y la placa Sudamericana originado por las variaciones en la dirección de convergencia es un factor importante en la formación de los mega controles estructurales propuesto. (Pardo-Casas \& Molnar, 1987), señalan que a la edad de $25.82 \mathrm{Ma}$ se produce un movimiento dextrógiro que origina una convergencia oblicua entre las placas (Nazca y Sudamericana), lo que se conserva hasta los 19.90 Ma y origino una componente de cizalla sinestral que se hizo evidente en el último evento de la fase Inca IV. Luego se ocasiona un giro antihorario de la placa de Nazca $\left(\mathrm{N} 75^{\circ} \mathrm{E}\right)$ manteniéndose así hasta la actualidad. Cabe mencionar que, debido al movimiento dextrógiro de la trayectoria de convergencia de la placa de Nazca, durante el Mioceno, la falla Punre-Canchis se reactiva, cambiando su sentido a un movimiento sinestral con un fuerte elemento de compresión y además con un elemento tensional producido en las zonas de inflexión de las fallas. Es importante también analizar el rol que jugo el ángulo de subducción que estuvo por alrededor de los $15^{\circ}$, para la localización de los yacimientos minerales, particularmente los localizados en el Corredor San PabloPorculla (90 km de la costa actual) y los del Corredor Michiquillay-Hualgayoc (140 Km de la costa actual), así como también la formación de las flexiones como las de Cajamarca y Cutervo, y la migración de los ejes magmáticos de Oeste a Este (Quispe et al., 2008).

Dentro del corredor estructural Chicama-Yanacocha, se han reconocido fallas y lineamientos de dirección NE que se consideran tensionales de las fallas de dirección andina (NO). Tomando en consideración una explicación genética se concluye que las fallas y lineamientos NE son rasgos estructurales complementarios de los grandes desplazamientos de las fallas de rumbo andino. Esta confluencia de fallas y lineamientos de orientación NE con fallas regionales de dirección andina configuran lugares favorables para el desarrollo de jogs estructurales, y para el emplazamiento de cuerpos magmáticos-hidrotermales. Cabe mencionar, que en la actualidad gran parte de estas fallas de dirección andina con componente strike slip se encuentran parcialmente cubiertas por los volcánicos del Neogeno (Calipuy y Huambos) y deformadas por fases tectónicas subsecuentes del Plio-Cuaternario); es donde se presentan interesantes oportunidades de zonas potenciales para reorientar nuestras exploraciones geológico-mineras.

Cabe mencionar, que dentro del Mega Sistema Estructural Cajamarca, controlado por fallas de rumbo andino y sus correspondientes antiandinas, algunos sectores pueden haber sido afectados por movimientos de compresión, otros de distensión (dilatación) e incluso oblicuo, provocando de esta manera, en diferentes tiempos zonas de debilitamiento cortical que facilitaron el desarrollo y emplazamiento de sistemas magmáticos del tipo volcánicos emergentes relacionados con fases de mineralización hidrotermal, vinculados con domos, diatremas, zonas de brechas e incluso la presencia de pórfidos.

\section{METODOS}

El presente documento es una investigación descriptiva ya que se basa en la descripción de las características de los fenómenos llegando a su caracterización, no experimental, haciendo uso de una metodología deductiva ya que partimos de lo general y llegamos a lo más específico.

Como punto de partida se realizó una recopilación bibliográfica de información disponible en INGEMMET y publicaciones internacionales sobre estratigrafía, magmatismo y controles estructurales del sector de estudio en base a la técnica documental. Información que finalmente luego de su análisis e interpretación permitió proponer un modelo estructural y definir controles estructurales. 


\section{RESULTADOS}

\subsection{Modelo Estructural Propuesto}

Se propone el Mega Sistema Estructural Cajamarca, el cual se sustenta en la existencia de tres sistemas de fracturas que pueden ser desarrolladas en una zona de fallas paralelas. Las estructuras mencionadas son las denominadas Riedel (R1 y R2), las fracturas tipo Y y las fracturas tipo P, es decir fracturas de tipo PYR. Sus dimensiones son kilométricas teniendo aproximadamente $200 \mathrm{~km}$ en su mayor dimensión lineal (NW) y $124 \mathrm{Km}$ en dirección NE.

Las superficies estructurales sintéticas R1 se configuran a un ángulo agudo $\left(15^{\circ}\right)$ con el plano principal de la zona de falla. Su distribución geométrica es en echelón (escalón), cabe mencionar, paralelas, pero separadas entre ellas en de la zona de falla. Las fallas antitéticas R2 son conjugadas con respecto a $\mathrm{R} 1$ y conforman un ángulo de $75^{\circ}$ en relación con el plano principal de la zona de falla. Asimismo, se tienen las fracturas Y, las cuales son paralelas a la envolvente y restringidas por el sentido de los esfuerzos de cizalla o corte. Relacionado con la evolución por la acción dinámica de la zona de falla puede manifestarse un tercer sistema de fracturas, que son los planos $\mathrm{P}$, conformando un ángulo agudo con el plano principal de la zona de falla (Figura 1).

Las fallas tienden a flexionarse en el espacio, debido a que el o los esfuerzos que las originan poseen una trayectoria determinada, lo cual da lugar al incremento de sectores que almacenan o liberan energía en zonas adyacentes a la o las fallas, es entonces en aquel lugar donde se generarán los espacios, que sin duda tendrán una mayor posibilidad de localizar mineralización importante. Las curvaturas que se dan en las fallas son importantes, puesto que se generan inflexiones en apertura, las cuales son donde se generan los espacios para que los fluidos hidrotermales pueden circular fácilmente. A escala regional, el emplazamiento de intrusiones bajo circunstancias relacionadas con esfuerzos tensionales, encuentran en las zonas de inflexión, una zona de apertura que favorecerá su intrusión.

Las zonas de apertura en las zonas de inflexión permiten la formación tanto de cuencas o depresiones (pullapart). Además, se generan también zonas de cierre en las cuales se puede dar lugar a levantamientos por efectos compresivos (pop-up) (Figura 2 y 3 ).

\subsection{Controles estructurales (modelo propuesto) durante la evolución tectónica andina}

Los Andes centrales, resultan de la subducción de las placas oceánicas Nazca (Farrallón), debajo de la placa continental Sudamericana.

El ciclo Andino comprende varias fases de sedimentación y etapas de deformación, las que fueron definidas por Gustav Steinmann (1929) como: Fase Peruana, la cual ocurrió durante el Cretácico superior y el Paleoceno, luego la Fase Incaica (Eoceno-Oligoceno), seguida por la Fase Quechua que ocurrió en el Mioceno y otras fases desarrolladas en el Plio-Cuaternario.

Según (Pardo-Casas \& Molnar, 1987), los periodos que dieron lugar a una convergencia rápida corresponden a la actividad de la Fase Inca y la Fase Quechua, siendo esta convergencia correspondiente a etapas de plegamiento y fallamiento inverso y oblicuo, que eran activos.

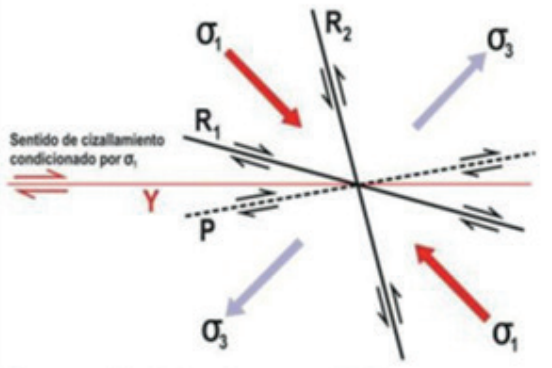

Generación de las fracturas PYR

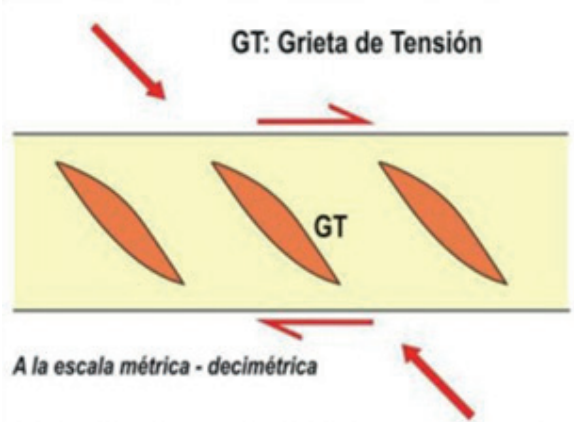

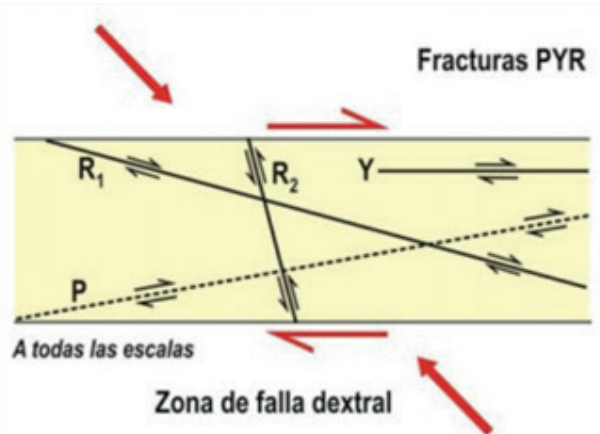

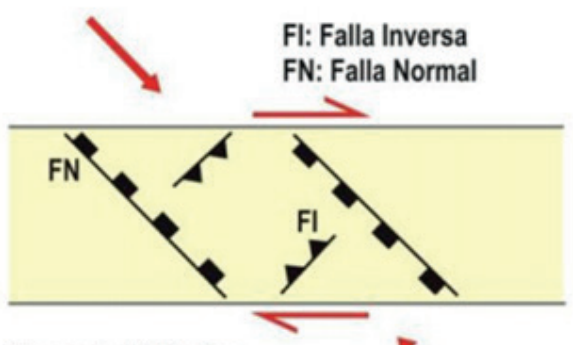

A la escala de kilómetros

Figura 1. Estructuras asociadas en una zona de falla por efecto del cizallamiento que podemos encontrar a distintas escalas. Adaptado de (Davis \& Reynolds, 1996) 
La compresión que predominó en el Cretácico superior (fase peruana), dio lugar a que la zona del arco volcánico inicie un levantamiento y separe la cuenca occidental del mar abierto, particularmente desarrollado en la cuenca occidental, sufriendo de esta manera dicha cuenca, cambios en los estados de esfuerzos regionales que la controlaban. En el tiempo, la compresión se fue desplazando hacia el este, ocasionando movimientos epirogénicos y deformación de la cuenca occidental, dando lugar a que las fallas de características regionales que gobernaban en la cuenca extensiva relacionada con fallas normales se activen con una cinemática inversa y tiendan a sustentar las cuencas sinorogénicas que se dan lugar al frente de los cabalgamientos. En el Eoceno, medio se da lugar la más importante deformación, produciéndose el acortamiento principal, un importante engrosamiento de la corteza y la deformación andina. La subducción es de manera oblicua generando así zonas permeables, visto en superficie como jogs estructurales dentro del Mega Sistema Estructural Cajamarca (Figura 3).

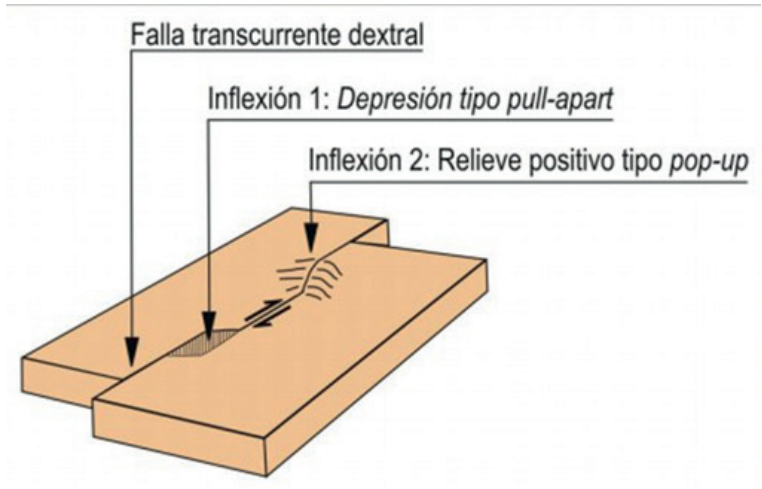

Figura 2. Contexto tipo pull apart y pop up. Adaptada de (Davis \& Reynolds, 1996))

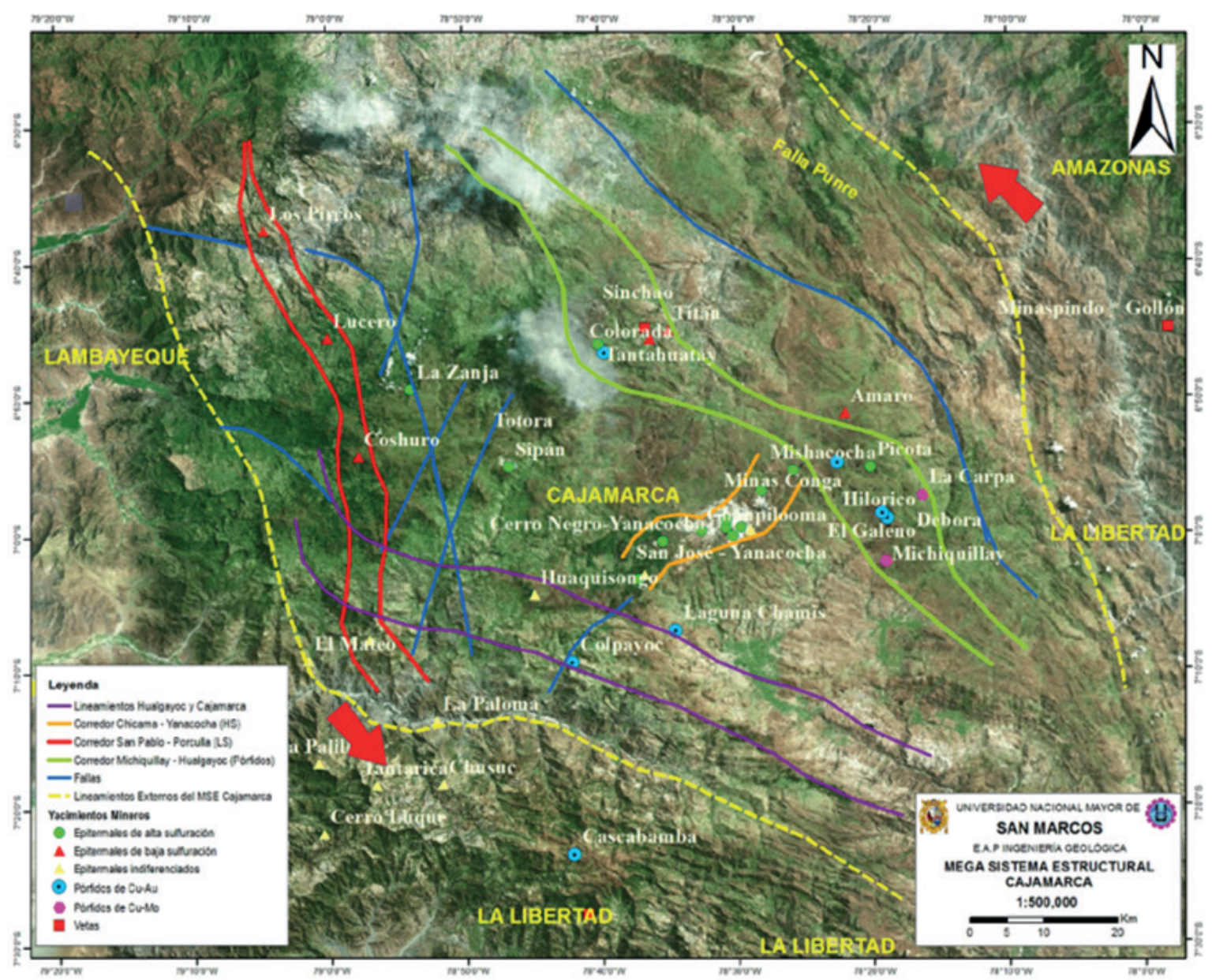

Figura 3. Mega Sistema Estructural Cajamarca (Tomado y Adaptado de Google Earth) 
Por otro lado, el acortamiento y engrosamiento también se puede explicar por el cambio en la dirección NorteSur de los Andes meridionales a una dirección Noroeste - Sureste actualmente en los Andes centrales, que se evidencia e inicia en el codo Arica-Santa Cruz y que luego generaron tres mega geobloques estructurales limitados por la flexión de Abancay y la flexión de Huancabamba, que a su vez internamente han generado geobloques estructurales producto de los diferentes efectos tectónicos producidos desde el Cretáceo hasta el Neogeno y donde se ha producido la conformación de mega estructuras de apertura y cierre que han orientado la mineralización, relacionados con fallas y lineamientos de dirección andina que de acuerdo a la velocidad de movimiento de los bloques y su resistencia litosférica conformaron estructuras tensionales y paralelas (Rabbia, 2012), (Figura 4).

La mínima resistencia en la corteza continental, en la cual se ha producido el engrosamiento durante el Cenozoico, favorece a una máxima deformación ante los eventos de subducción, debido a que gran parte se encuentra en una zona denominada frágil hasta los $35 \mathrm{~km}$ aproximadamente (Rabbia, 2012), (Figura 4).

En particular en la fase peruana, ocurren eventos de deformación por efecto de la compresión, que afecta a la Costa, Cordillera Occidental y también al Altiplano. En el frente andino se originaron amplias zonas de debilidad por intenso fracturamiento, lo que permitió generar el espacio para que ascienda y se localice el Batolito de la costa, cortando de esta manera las secuencias plegadas del Mesozoico.

La fase Incaica es la más relevante del ciclo andino, principalmente aateñea tanto al Norte como al Centro del Perú. Durante el Paleoceno superior-Oligoceno se da un reinicio de la alta convergencia de placas, en donde los controles estructurales de los sistemas en el norte (debido a su transformación en el tiempo, distribución espacial y sus semejanzas geoquímicas), implican un evidente control estructural del sistema de fallas NO-SE Punre-CanchisMagistral, con registros de un movimiento inicial dextral oblicuo, produciéndose magmatismo asociado al primer evento, que da lugar a la intrusión de sistemas pórfidos en Cajamarca (Rivera \& Santisteban, 2008), que cortan a las facies plegadas del Mesozoico.

La fase Quechua corresponde a eventos compresivos que generaron zonas de inflexión y fallas conjugadas en las cuales se formaron aberturas producto de la tensión, permitiendo la circulación de fluidos magmáticoshidrotermales, cambiando el movimiento a sinestral compresivo y oblicuo durante esta fase.

Aparentemente, los movimientos dextral y sinestral del sistema de fallas, estarían relacionados con el cambio de orientación de la convergencia de la placa de Nazca, ya que previamente al Mioceno la placa de Nazca/Farallón tenía una orientación de convergencia noreste y la convergencia alternadamente intervenía entre las zonas altas y bajas, y durante el Mioceno temprano la rotación fue en sentido horario del ángulo de convergencia de la placa de Nazca, aproximadamente $20^{\circ}$ (Pardo-Casas \& Molnar, 1987), (Figura 5).

Por otro lado, de acuerdo con el grosor de la corteza, los arcos magmáticos pueden ser divididos en arcos inmaduros (corteza continental delgada 25-40 km), en la cual aparece anfibolita (roca metamórfica con anfíbol y plagioclasa). Mientras que los Arcos maduros (corteza continental gruesa mayor a $45 \mathrm{~km}$ ) existe eclogita (el anfíbol $\mathrm{u}$ hornblenda se rompe generando piroxeno con liberación de H2O, y también granate) (Montano, 2010).

La tendencia de las tierras raras es usualmente referida como patrones de tierras raras y son de considerable interés petrológico. Dicha tendencia es presentada de acuerdo con las concentraciones de tierras raras normalizadas a condrito y expresadas en logaritmo de base 10. Los minerales predominantes en la corteza tienen un efecto característico en el patrón de tierras raras del fundido. Para cada espesor de la corteza existen tipos de yacimientos asociados que son inferidos de acuerdo con el patrón de comportamiento de las tierras raras.
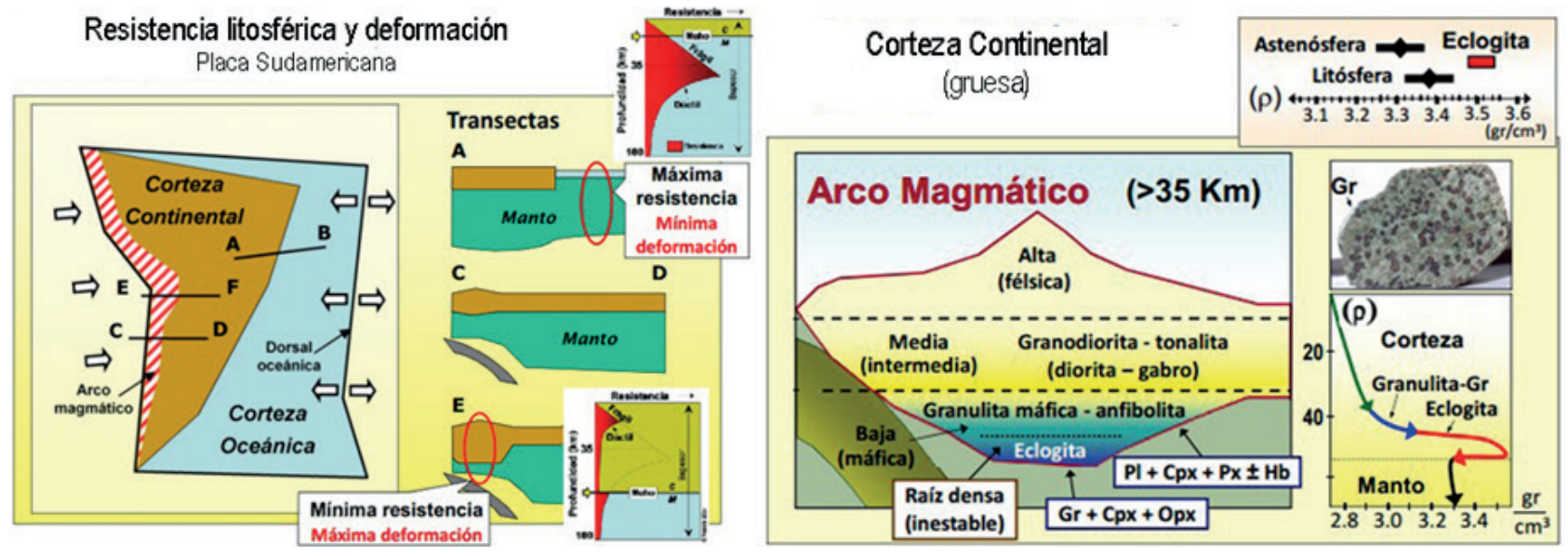

Figura 4. Resistencia Litosférica y deformación (Rabbia, 2012) y Engrosamiento cortical durante el Cenozoico (Rabbia, 2012) 
Variación de la tasa de convergencia en los últimos $60 \mathrm{Ma}$

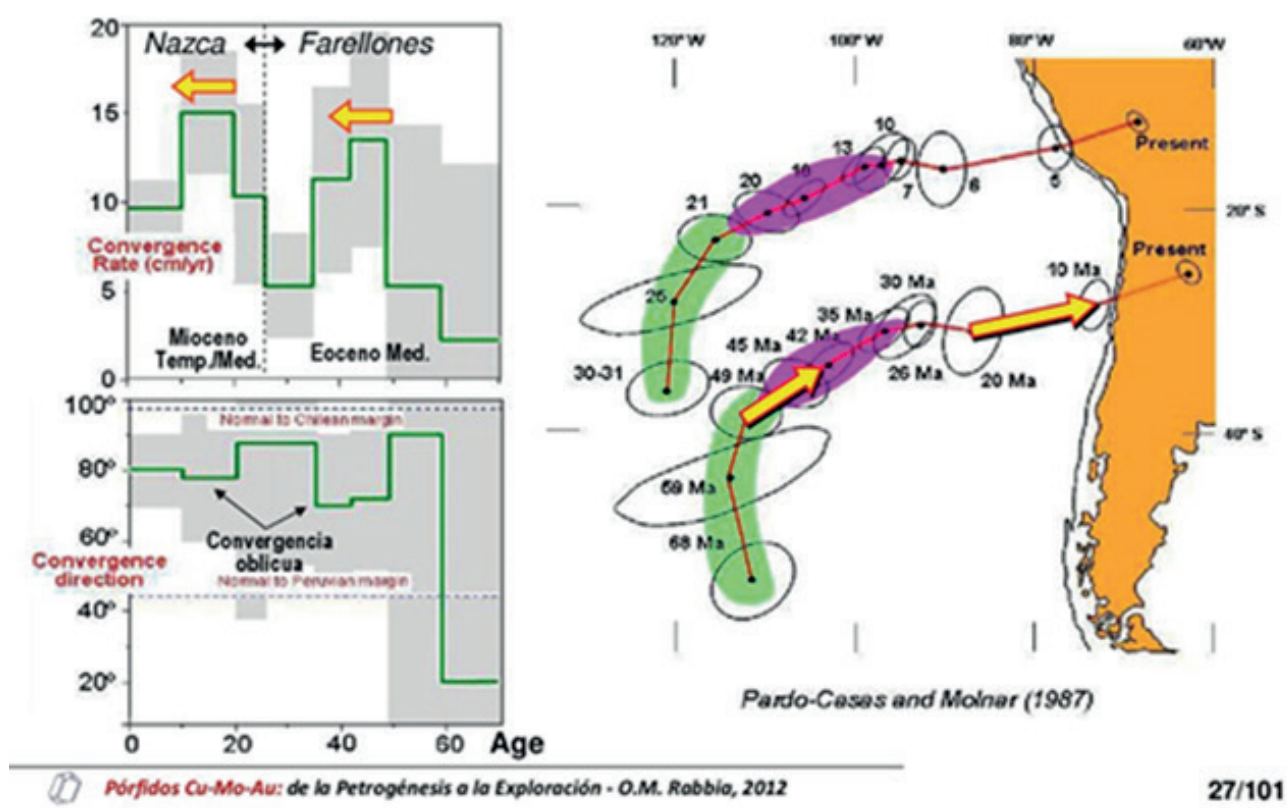

Figura 5. Placa de Nazca (Farallones) y la evolución de su ubicación a través de los últimos 60 ma.

(Pardo-Casas \& Molnar, 1987)

Las ratios de $\mathrm{La} / \mathrm{Sm}$ y $\mathrm{Sm} / \mathrm{Yb}$ muestran el comportamiento de las tierras raras livianas y las tierras raras pesadas respectivamente. El incremento de las ratios de tierras raras pesadas es atribuido al incremento de anfíboles y granates como minerales residuales.

Por lo tanto, una interpretación sugiere que un magma que cristalizo en una corteza controlada por mineral residual anfíbol sin clinopiroxeno ni granate, probablemente cristalizo durante un engrosamiento de la corteza, pudiendo dar origen a depósitos de pórfidos $\mathrm{Cu}-\mathrm{Mo}$.

Finalmente, los rasgos morfoestructurales regionales, nos ayudan a comprender la evolución y característica geodinámica de Perú, la cual está controlada por los siguientes rasgos tectónicos: la Fosa peruano-chilena, la Cordillera Andina, la Dorsal de Nazca, la Cadena Volcánica y los diferentes Sistemas de fallas distribuidas a lo largo del interior del país. Adicionalmente, tomamos en cuenta que, en este sector norte del país, se ha desarrollado una zona de convergencia oblicua de las placas en la zona de subducción, así como la variación del ángulo de subducción $\left(15^{\circ}\right)$, como rasgos importantes para la creación de depósitos minerales. También consideramos importante analizar que la posición Norte-Sur de los andes meridionales, ha cambiado con respecto a la de los andes centrales siendo esta Noroeste, esto se evidencia de manera contundente con la presencia del codo Arica-Santa Cruz y cuyos efectos son notorios en el Perú, Bolivia y Brasil. Particularmente en Perú, se han formado tres mega-geobloques estructurales separados al Sur por la flexión de Abancay y al Norte por la flexión de Huancabamba. Estos movimientos hacia el Oeste en los andes centrales (fases Inca y Quechua) son los que han ayudado también a formar enormes controles estructurales de dirección andina a modo de compartimientos de fallas de desplazamiento de rumbo desarrollados en regímenes de cizalla que morfológicamente tienen la geometría de un mega-cimoides, como el que proponemos en el presente y que no solo se observa en Cajamarca, sino también se repite al sur en la Libertad (Laguna Norte), al norte en Piura (Tambo Grande), e incluso Ancash (Pierina) con similar comportamiento tectónico estructural, con generación de jogs estructurales adecuados para albergar mineralización y zonas de cierre generados por movimientos de tipo strike slip, que forman sistemas conjugados de dimensiones kilométricas, dentro de cuencas de tipo pull apart que requieren de una mayor y continua investigación para su precisa definición.

\section{DISCUSIÓN}

El desarrollo de sistemas de cizalla en la tectónica Andina si bien ha influenciado en gran parte la localización de los controles estructurales y la mineralización en el norte del Perú aún necesita mayor investigación, se propone que tienen su fundamento en los sistemas de fallas regionales de dirección andina y que por razones de su convergencia oblicua entre las placas de Nazca y Sudamericana tiene un factor de rumbo, formando así fallas de desplazamiento de rumbo tipo strike slip, lo que es claramente observable en los corredores estructurales San Pablo-Porculla (Enríquez et al., 2000) y Michiquillay - Hualgayoc. Con respecto a las del corredor Chicama - Yanacocha, de tipo transversal (NE) se las explica considerando que estas son expresiones estructurales complementaria de las fallas de rumbo andino (Rivera \& Santisteban, 2008).

Los conceptos presentados por los autores son válidos y se encuentra debidamente sustentados, razón por la cual 
consideramos importante darle un marco estructural de conjunto. Es la razón por la que sugerimos la denominación de Mega Sistema Estructural Cajamarca, que involucra una configuración de conjunto estructural lo que significa un relacionamiento en sí mismo y que a su vez define un marco estructural con límites que delimitan dicho conjunto.

\section{CONCLUSIONES}

La mayor producción de magmas félsicos se da como consecuencia del engrosamiento cortical, lo cual es favorable para la formación de grandes sistemas de pórfidos.

La inversión tectónica durante el cenozoico y una resistencia mínima de la corteza, fueron factores importantes en el engrosamiento cortical. Durante esta inversión las fallas normales cambiaron a inversas.

La subducción oblicua ocasiona que las grandes fallas regionales de rumbo andino generen una componente de rumbo asociada, que dan lugar a dominios estructurales que orientan la distribución espacial de los depósitos minerales y además favorecen a zonas permeables en la cuales existe mineralización que se ven representadas en superficie como jogs estructurales que orientaron el magmatismo a superficie (domos, calderas).

La fase Quechua correspondería al evento más importante para la formación de yacimientos en el Mega Sistema Estructural Cajamarca, dado que esta fase es compresiva y de mayor engrosamiento cortical, que permitió la generación de zonas de inflexión en las cuales se formaron aberturas producto de la tensión, permitiendo la circulación de fluidos magmáticos-hidrotermales en el área de estudio.

Observado los lineamientos y las estructuras en las imágenes satelitales se evidencia la existencia del sistema estructural propuesto: Mega Sistema Estructural Cajamarca, será necesario realizar más estudios de campo con la finalidad de enriquecer y afianzar lo propuesto, será necesario tomar lecturas de paleomagnetismo, dataciones, litogeoquímica entre otras.

Cuando las dorsales se encuentran muy próximas como son los casos de Carnegie, Grijalva y Sarmiento se produjo una subducción de aproximadamente $30^{\circ}$; sin embargo, cuando se encuentran muy espaciadas como es el caso entre las dorsales de Sarmiento y Nazca, en la que se ubica la zona Norte del Perú, se producirá una subducción anómala de aproximadamente $10^{\circ}$ hacia el continente para luego desplegarse como una subducción horizontal por debajo de los andes. La mineralización en la zona Norte del Perú puede también deberse a esta variación del ángulo de subducción de la placa, así como a la oblicuidad de la subducción, migración de los ejes magmáticos entre otros, que conformaron mega estructuras estructurales como la propuesta.

\section{AGRADECIMIENTOS}

Agradecemos a la Escuela Académico profesional de Ingeniería Geológica, en la persona de su director $\mathrm{Mg}$. Ciro Bedia Guillen por su apoyo en habernos brindado las facilidades de poder hacer esta investigación, así como al profesor Ing. Jesús Alberto Torres Guerra por sus consejos y aliento constante en la elaboración del presente.

\section{REFERENCIAS}

Davies, R. C. I. (2006). Tectonic, Magmatic and Metallogenic Evolution of The Cajamarca Mining district, Northern Peru (Abstract and Introduction). PhD Thesis, 1-20. https:// researchonline.jcu.edu.au/10/

Davies, R. C., \& Williams, P. J. (2005). The El Galeno and Michiquillay porphyry $\mathrm{Cu}-\mathrm{Au}-\mathrm{Mo}$ deposits: Geological descriptions and comparison of Miocene porphyry systems in the Cajamarca district, northern Peru. Mineralium Deposita, 40(5), 598-616. https://doi.org/10.1007/s00126005-0026-6

Davis, G. H., \& Reynolds, S. (1996). Structural Geology of Rocks and Regions - George H. Davis, Stephen J. Reynolds, Charles F. Kluth - Google Libros. https://books.google.com. pe/books?hl=es\&lr=\&id=EYzzOKLRT-8C\&oi=fnd\&pg=P A2\&dq=Davis,+ G.H. + and + Reynolds,+ S. $+(1996)+$ Structu ral + Geology + of + Rocks + and + Regions. +2 nd. + Edition. + Ed .+John+Wiley+\%26 +Sons ,+Inc.\&ots=2B5ZYnIirl\&sig= vS2uqQVH6pw6zNMCp6qAzRgCH8k\#v=onepage\&

Enríquez, J. a., Rodríguez, O., \& Rodríguez, R. (2000). Características estructurales y estratigráficas de los yacimientos epitermales de baja sulfuración en el corredor estructural San Pablo - Porculla. XIII Congreso Peruano de Geología. Resúmenes Extendidos. Sociedad Geológica Del Perú., Wilson, 724-727. https://www.academia. edu/8651733/GEOLOGÍA DE LOS YACIMIENTOS EPITERMALES_DEL_TIPO_BAJA_SULFURACIÓN EN_EL_CORREDOR_ESTRŪCTURAL_SAN_PABLO_PORCULLLA_NORTE_DEL_PERÚ

Mamani, M., Worner, G., \& Sempere, T. (2010). Geochemical variations in igneous rocks of the Central Andean orocline (13 $\mathrm{S}$ to $18 \mathrm{~S}$ ): Tracing crustal thickening and magma generation through time and space. Geological Society of America Bulletin, 122(1-2), 162-182. https://doi. org/10.1130/B26538.1

Montano, H. (2010). Caracterización geoquímica de magmas y su relación con la formación de yacimientos en el norte del Perú. October. http://cybertesis.uni.edu.pe/handle/ uni/8975

Pardo-Casas, F., \& Molnar, P. (1987). Relative motion of the Nazca (Farallon) and South American Plates since Late Cretaceous time. Tectonics, 6(3), 233-248. https://doi. org/10.1029/TC006i003p00233

Quispe, J., Carlotto, V., Acosta, J., Macharé, J., Chirif, H., Rivera, R., Romero, D., Huanacuni, D., \& Rodriguez, R. (2008). Mapa metalogenético del Perú 2008: herramienta esencial para las las exploraciones mineras. XIV Congreso Peruano de Geología y XIII Congreso Latinoamericano de Geología, Soc Geol Perú. https://es.calameo.com/ $\mathrm{read} / 000820129 \mathrm{a} 7442 \mathrm{f3edfe} 7$

Rabbia, O. (2012). Pórfidos Cu-Mo-Au: de la Petrogénesis a la Exploración. Universidad Nacional de Río Cuarto. Departamento de Geología. Curso Intensivo de Posgrado. https://es.scribd.com/document/373671667/Petrogenesisde-Porfidos-de-Cu-Dr-Osvaldo-Rabbia-pdf 
Rivera, R., Quispe, J., Rodriguez, I., \& Villarreal, E. (2008). Comparación entre los sistemas porfiriticos de Cu-Mo y $\mathrm{Cu}-\mathrm{Au}$ del Norte del Perú (Región Cajamarca). INGEMMET. moz-extension://af9c8ffb6ba6-4457-980e-9fee 00c031c9/enhanced-reader. html?openApp\&pdf=https $\% 3 \mathrm{~A} \% 2 \mathrm{~F} \% 2 \mathrm{Fwww}$.ingemmet. gob.pe\%2Fdocuments\%2F73138\%2F202784\%2FP12 Expo_Congreso_2008.pdf\%2F8a6abb14-f977-4deb-80ff$\mathrm{d} 07 \mathrm{~b} \overline{\mathrm{d}} 7 \mathrm{~b} 743 \mathrm{c} 0$

Rivera, R., \& Santisteban, A. (2008). Control Estructural, Producción y Reservas en las Franjas Metalogenéticas del Norte del Perú(Región de Cajamarca). Dirección de Recursos Minerales y Energéticos de INGEMMET. moz-extension:// af9c8ffb-6ba6-4457-980e-9fee00c031c9/enhanced-reader. html?openApp\&pdf $=$ https $\% 3 \mathrm{~A} \% 2 \mathrm{~F} \% 2 \mathrm{Fwww}$.ingemmet. gob.pe\%2Fdocuments\%2F73138\%2F202784\%2F003 2011_Articulo_2011_VIII_ProExplo_Control_Estructural _Produccion_Reservas_Franjas_Metalogeneticas_nor
Skinner, B. J., \& Benavides-Cáceres, V. (1999). Geology and Ore Deposits of the Central Andes. In Geology and Ore Deposits of the Central Andes (Special Pu). Society of Economic Geologists. https://doi.org/https://doi.org/10.5382/SP.07.03

Soler, P. (1991). Contribution à l'étude du magmatisme associé aux marges actives. Pétrographie, géochimie et géochimie isotopique du magamtisme crétacé à pliocène le long d'une transversale des Andes du Pérou central. Implications géodynamiques et métallogéniques. [Paris 6]. In http://www. theses.fr. http://www.theses.fr/1991PA066637

Steinman, G. (1929). Geologie von Penú. Nature, 124(3138), 943-943. https://doi.org/10.1038/124943b0 